\title{
Assessing the feasibility of using the short physical performance battery to measure function in the immediate postoperative period after total knee replacement
}

\author{
Rene Przkora (1), Kimberly Sibille (1,2), Sandra Victor (1), Matthew Meroney (1), \\ Christiaan Leeuwenburgh (2,3), Anna Gardner (4), Terrie Vasilopoulos (1,5), Hari K. \\ Parvataneni (5)
}

(1) Department of Anesthesiology, University of Florida College of Medicine, Gainesville, Florida; (2) Department of Aging \& Geriatric Research, University of Florida College of Medicine, Gainesville, Florida; (3) Department of Anatomy and Cell Biology, University of Florida College of Medicine, Gainesville, Florida; (4) Department of Applied Physiology \& Kinesiology, University of Florida College of Health \& Human Performance, Gainesville, Florida; (5) Department of Orthopaedics and Rehabilitation, University of Florida College of Medicine, Gainesville, Florida.

This article is distributed under the terms of the Creative Commons Attribution Noncommercial License (CC BY-NC 4.0) which permits any noncommercial use, distribution, and reproduction in any medium, provided the original author(s) and source are credited.

\begin{abstract}
Measurements of physical function after total knee arthroplasty (TKA) are uncertain and require investments for special equipment, space, and staff. Therefore, we evaluated the Short Physical Performance Battery (SPPB) 4 to 6 weeks preoperatively and 2 weeks following TKA as this test battery addresses lower extremity strength, coordination, and balance, without additional special investments. For context, we also employed the Six-Minute Walk test (6MWT) and peak torque knee extension. Our analysis consisted of three women and one man, with average ages of $68.3 \pm 3.9$ years. Patients showed a decrease in all functional measurements: SPPB (mean change: $-4.8 \pm 1.9,44 \%$ decrease), $6 \mathrm{MWT}(-0.37 \pm .21 \mathrm{~m} / \mathrm{s}, 34 \%$ decrease), and peak torque $(-11.8 \pm 8.8 \% \mathrm{~s}, 68 \%$ decrease). Our findings suggest the SPPB is feasible and can be integrated easily in daily clinical practice.
\end{abstract}

Key Words: knee joint; knee replacement arthroplasty; outcome assessment; pain; physical function performance

Eur J Transl Myol 31 (2): 9673, 2021 doi: 10.4081/ejtm.2021.9673

Older adults are the fastest-growing patient population and aging is associated with increased degenerative changes in major joints, leading to activity-limiting pain and loss of function. Data indicate that 60 million Americans have osteoarthritis (OA), a number that is expected to double over the next decade. ${ }^{1-3}$ Knee OA is the leading cause of disability among older adults. ${ }^{1}$ The aging population and the obesity epidemic are established risk factors for OA, especially for knee OA. With the population older than 65 years expected to nearly double by 2030, a significant increase in OA prevalence is anticipated, with an unprecedented demand on the health system. ${ }^{2}$ The knee joint is the largest joint of the human body and is vital for all normal daily activities and independence. Knee OA is characterized by significant pain, progressive joint space destruction, and loss of muscle mass and function. ${ }^{3-5}$ The etiology of OA of the knee joint is multifactorial, with a combination of genetic predisposition, history of trauma, habitus and physical activity, and strength, especially of the knee extensor muscles. ${ }^{6}$ A significant number of patients will require a total knee arthroplasty (TKA). ${ }^{3}$ TKA is a major surgery associated with significant morbidity and mortality. ${ }^{7}$ The surgical procedure is performed under anesthesia and can require hospital admission for 1 to 2 days or more, depending on the clinical course. Some patients require prolonged stay in an assisted care facility or additional care during the recovery period. Postoperative pain is significant and Acute Pain Services are frequently involved during the hospital stay. ${ }^{8}$ Early physical therapy and ambulation is the primary goal in the postoperative course. In 2009, 620,000 knee joint arthroplasties were performed in the US, a number that has nearly doubled compared to a decade ago, ${ }^{9}$ and the number of annual TKA procedures in the US is projected to increase to nearly 3.5 million by the year $2030 .{ }^{10}$ The indication for a TKA is the need to reduce pain and increase mobility. However, up to $20 \%$ of patients have reported being dissatisfied with their postoperative outcome, ${ }^{11}$ and chronic pain has been reported by up to $35 \%$ of patients who underwent a TKA. ${ }^{12}$ Additionally, 
loss of function and muscle mass continues after surgery, specifically in the elderly, and is characterized by very poor recovery. Several tests are established to evaluate physical function and strength in patients undergoing a TKA, such as the Six-Minute Walk test (6MWT) and peak torque knee extension measurements. ${ }^{13,14}$ However, these tests are elaborative and frequently require special equipment, space, and staff. The purpose of this investigation was to determine the feasibility and acceptability of the Short Physical Performance Battery (SPPB) as a clinically applicable, simple, and objective test to evaluate physical function in older patients undergoing an elective TKA. The SPPB may serve as a brief and more convenient measurement when compared to conventional, more elaborate tests that are difficult to perform in regular practice, such as the 6MWT and peak torque knee extension.

\section{Materials and Methods}

Four patients were included in the evaluation of the feasibility of the SPPB to measure pre- and immediate postoperative physical function after an elective, unilateral TKA in older patients. Our study was approved by the Institutional Review Board and participants provided written informed consent. We tested the following parameters 4 to 6 weeks preoperatively and 2 weeks postoperatively: the SPPB, the 6MWT, leg strength of the operative leg (peak torque knee extension), and pain score (numerical rating scale). The SPPB includes three objective tests of lower body function: 1) a timed 4-m walk; 2) timed, repetitive chair stands; and 3) a hierarchical test of standing balance. The SPPB summary score is created by adding the three individual test items according to previously established criteria. There is a potential range of 0 to 12 , with higher scores indicating better lower body function. ${ }^{15} \mathrm{~A}$ 1-point improvement in the SPPB summary score has been indicated as clinically meaningful and correlated well with increases in overall activity and survival. ${ }^{15}$ The 6MWT: Subjects walk back and forth along a 100-ft hallway for 6 minutes after standardized instructions to complete as many laps as possible. The distance covered in 6 minutes is recorded. ${ }^{13}$ Strength of the quadriceps extensor muscles (peak torque) was tested using a dynamometer (Biodex, Shirley, NY). The subject was seated on the Biodex seat and strapped in around their shoulders/torso and knees to keep them in position. The operative leg was attached to the knee attachment of the dynamometer with a padded strap at the ankle. Subjects were instructed to perform a sufficient number of submaximal repetitions and then three maximal repetitions. The quadriceps femoris peak torques and corresponding knee angles were measured at a speed of $60 \%$ sec. Each subject performed, with verbal encouragement, three maximal voluntary repetitions. The best peak torque was recorded. ${ }^{14}$

Pain intensity was measured using the numerical rating scale (0-10), with 0 indicating no pain and 10 indicating worst pain. The pain intensity rating was obtained at the beginning of each appointment visit. Anesthetic and postoperative management was similar among patients; all surgeries were performed under a spinal anesthetic and regional anesthesia including a femoral nerve catheter and sciatic nerve single-injection regional anesthetic.

\section{Results}

Data on four individuals were analyzed (three women/one man). The average age was $68.3 \pm 3.9$ years and body mass index was $33.3 \pm 6$. Table 1 reports demographics of the sample. Postoperatively, all patients showed a decrease in all functional measurements: SBBP (mean change: $-4.8,95 \% \mathrm{CI}:-7.7,-1.7,44 \%$ decrease), 6MWT $(-0.37 \mathrm{~m} / \mathrm{s}, 95 \% \mathrm{CI}:-0.89,0.16,34 \%$ decrease), and peak torque extension $(-11.8 \%$ s, $95 \% \mathrm{CI}:-90.6,67.0$, $68 \%$ decrease). Patients also showed a $32 \%$ increase in pain compared to baseline. Results are summarized in Figure 1.

\section{Discussion}

The purpose of the study was to evaluate the feasibility and acceptability of the SPPB as a clinically applicable, simple, and objective test to evaluate physical function in older patients undergoing an elective TKA. Our pilot study demonstrated that the completion of the SPPB was feasible and acceptable prior to and within 2 weeks following the TKA procedure. There was no special investment necessary for the SPPB. Additionally, preliminary findings suggest across all measurements a clinically significant decline in physical function and strength of the lower extremity and an increase in pain postoperatively following elective total knee replacement surgery. With the increase in the aging population, the need for knee joint replacement surgery will continue to grow. The targeted benefits of TKA include reducing pain and increasing mobility and function. Studies comparing patients who underwent a TKA to healthy

Table 1. Sample demographics

\begin{tabular}{lc}
\hline \multicolumn{1}{c}{ Measurement } & $\mathbf{N}=\mathbf{4}$ \\
\hline $\begin{array}{l}\text { Age, mean year } \pm \text { SD } \\
\text { Gender, } \mathbf{n}\end{array}$ & $68.3 \pm 3.9$ \\
$\begin{array}{l}\text { Female } \\
\text { Male }\end{array}$ & 3 \\
Ethnicity, $\mathbf{n}$ & 1 \\
Hispanic & \\
Non-Hispanic & 1 \\
Race, $\mathbf{n}$ & 3 \\
White & \\
Black/African-American & 3 \\
Highest level of education, $\mathbf{n}$ & 1 \\
College graduate & \\
Some college, but not degree completion & 1 \\
\hline
\end{tabular}



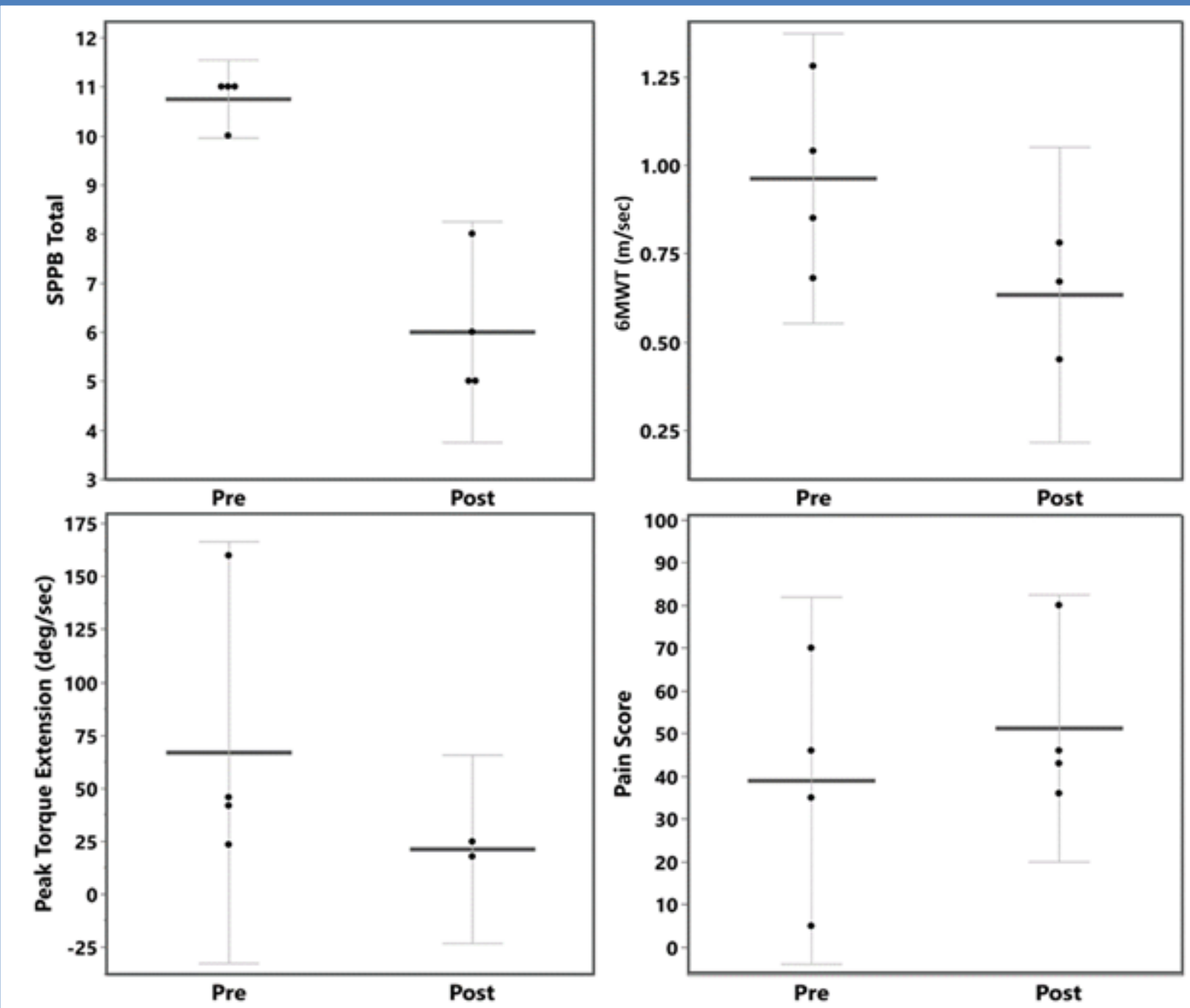

Fig 1. Pre- and post-values for Short Physical Performance Battery (SPPB), Six-Minute Walk Test (6MWT), peak torque extension, and pain score. Solid horizontal lines represent mean values, dots represent individual patient values, and error bars represent $95 \%$ confidence intervals.

age-matched control subjects demonstrated that patients who underwent a TKA remained weaker in quadricep strength throughout study periods beyond 3 years. Identifying brief and easy to administer physical functioning measurements that can be adapted in the clinic to evaluate decline or progress will improve patient management and outcomes following TKA. As anticipated, our pilot study demonstrated a significant decline in objective physical parameters in the postoperative period. ${ }^{16,17}$ The SPPB has been studied across a wide range of populations; however, it is not typically included in the evaluation of physical performance following TKA. ${ }^{15-19}$ As a postoperative functional outcome measurement, the SPPB has mainly been investigated and used in patients undergoing cardiac or pulmonary surgery. A low SPPB score has been associated with adverse surgical outcomes. In patients undergoing mitral valve replacement surgery, a SPPB score below 12 was a predictor of unplanned readmissions. ${ }^{18}$ A SPPB score below 10 was associated with higher postoperative pulmonary complications in elderly patients undergoing lung resection. ${ }^{19}$ Przkora et al. demonstrated the utility of the SPPB in ambulatory patients undergoing pain procedures for degenerative spine disease. ${ }^{20}$ Our findings suggest that the collection of the SPPB in the perioperative setting in patients following lower extremity joint replacement surgery is feasible and acceptable. Patients had a mean SPPB of $10.75 \pm 0.5$ prior to surgery, which declined to a mean of $6 \pm 1.4$ postoperatively, a $44 \%$ decrease ( 3 to 4 points). The decrease in the SPPB in the postoperative period also occurred in conjunction with declines in the 6MWT and knee extension. Future investigations with a larger sample size are needed to confirm the sensitivity of the SPPB to meaningful clinical changes in patients undergoing TKA and comparative utility with recognized standard of care instruments. In conclusion, our pilot study indicates that completing the SPPB presurgically 
and within a 2-week postoperative period is feasible and acceptable. Given the importance of monitoring the physical function of patients undergoing TKA following the surgical procedure to optimize physical function, the SPPB might serve as a simple and easy to administer tool in the clinical setting to monitor patient progress and refer for necessary services as needed.

\section{List of acronyms}

OA - osteoarthritis

TKA - total knee arthroplasty

SPPB - Short Physical Performance Battery

6MWT - Six-Minute Walk test

\section{Authors contributions}

All authors made substantial contributions to the conception, design, and analysis and interpretation of data for this work. All authors contributed substantially to the drafting and critical revision of the manuscript, provided final approval of the version submitted, and agree to be held accountable for all aspects of the work.

\section{Acknowledgments}

None.

\section{Funding}

This work was sponsored by a grant from the Clinical and Translational Science Institute of the University of Florida (R.P.).

\section{Conflict of Interest}

The author declares no competing interests.

\section{Ethical Publication Statement}

We confirm that we have read the Journal's position on the issues involved in ethical publication and affirm that this report is consistent with those guidelines.

\section{Corresponding Author}

Rene Przkora, MD, PhD, Department of Anesthesiology, University of Florida College of Medicine, 1600 SW Archer Road, PO Box 100254, Gainesville, FL 32610. Phone: +1-352-265-7777; Fax: +1-352-392-7029

ORCID iD: 0000-0003-4298-4602

E-mail: rprzkora@anest.ufl.edu

\section{E-mails and ORCID iDs of Coauthors}

Kimberly Sibille: ksibille@ufl.edu ORCID iD: 0000-0002-8313-0024

Sandra Victor: svictor@anest.ufl.edu

ORCID iD: 0000-0003-4572-3619

Matthew Meroney: mmeroney@anest.ufl.edu

ORCID iD: 0000-0001-9884-0347

Christiaan Leeuwenburgh: cleeuwen@ufl.edu;

ORCID iD: 0000-0003-0826-4257

Anna Gardner: akgardner@ufl.edu

ORCID iD: 0000-0001-7429-5081

Terrie Vasilopoulos: tvasilopoulos@anest.ufl.edu

ORCID iD:0000-0002-8066-2703
Hari K. Parvataneni: parvahk@ortho.ufl.edu ORCID iD: 0000-0001-5547-405X

\section{References}

1. Neogi T, Zhang Y. Epidemiology of osteoarthritis. Rheum Dis Clin North Am. 2013 Feb;39(1):1-19. doi: 10.1016/j.rdc.2012.10.004. Epub 2012 Nov 10.

2. Centers for Disease Control and Prevention (CDC). Prevalence and most common causes of disability among adults - United States (2005). MMWR Morb Mortal Wkly Rep 2009;58:421-6.

3. Nelson AE, Allen KD, Golightly YM, Goode AP, Jordan JM. A systematic review of recommendations and guidelines for the management of osteoarthritis: The chronic osteoarthritis management initiative of the U.S. bone and joint initiative. Semin Arthritis Rheum. 2014 Jun;43(6):701-12. doi: 10.1016/j.semarthrit. 2013.11.012. Epub 2013 Dec 4. PMID: 24387819.

4. van Dijk GM, Dekker J, Veenhof C, van den Ende $\mathrm{CH}$; Carpa Study Group. Course of functional status and pain in osteoarthritis of the hip or knee: a systematic review of the literature. Arthritis Rheum. 2006 Oct 15;55(5):779-85. doi: 10.1002/art.22244.

5. Slemenda C, Brandt KD, Heilman DK, Mazzuca S, Braunstein EM, Katz BP, Wolinsky FD. Quadriceps weakness and osteoarthritis of the knee. Ann Intern Med. 1997 Jul 15;127(2):97-104. doi: 10.7326/0003-4819-127-2-199707150-00001.

6. Øiestad BE, Juhl CB, Eitzen I, Thorlund JB. Knee extensor muscle weakness is a risk factor for development of knee osteoarthritis. A systematic review and meta-analysis. Osteoarthritis Cartilage. 2015 Feb;23(2):171-7. doi: 10.1016/j.joca.2014. 10.008.

7. Ravi B, Escott B, Shah PS, Jenkinson R, Chahal J, Bogoch E, Kreder H, Hawker G. A systematic review and meta-analysis comparing complications following total joint arthroplasty for rheumatoid arthritis versus for osteoarthritis. Arthritis Rheum. 2012 Dec;64(12):3839-49. doi: 10.1002/art.37690.

8. McCartney CJ, Nelligan K. Postoperative pain management after total knee arthroplasty in elderly patients: treatment options. Drugs Aging. 2014 Feb;31(2):83-91. doi: 10.1007/s40266-013-0148-y.

9. Weinstein AM, Rome BN, Reichmann WM, Collins JE, Burbine SA, Thornhill TS, Wright J, Katz JN, Losina E. Estimating the burden of total knee replacement in the United States. J Bone Joint Surg Am. 2013 Mar 6;95(5):385-92. doi: 10.2106/JBJS.L.00206.

10. Kurtz S, Ong K, Lau E, Mowat F, Halpern M. Projections of primary and revision hip and knee arthroplasty in the United States from 2005 to 2030. J Bone Joint Surg Am. 2007 Apr;89(4):780-5. doi: 10.2106/JBJS.F.00222.

11. Hofmann S, Seitlinger G, Djahani O, Pietsch M. The painful knee after TKA: a diagnostic algorithm 
for failure analysis. Knee Surg Sports Traumatol Arthrosc. 2011 Sep;19(9):1442-52. doi: 10.1007/s00167-011-1634-6. Epub 2011 Aug 6.

12. Beswick AD, Wylde V, Gooberman-Hill R, Blom A, Dieppe P. What proportion of patients report long-term pain after total hip or knee replacement for osteoarthritis? A systematic review of prospective studies in unselected patients. BMJ Open. 2012 Feb 22;2(1):e000435. doi: 10.1136/bmjopen-2011-000435.

13. ATS Committee on Proficiency Standards for Clinical Pulmonary Function Laboratories. ATS statement: guidelines for the six-minute walk test. Am J Respir Crit Care Med. 2002;166:111-7. doi: 10.1164/ajrccm.166.1.at1102

14. Borges O. Isometric and isokinetic knee extension and flexion torque in men and women aged 20-70. Scand J Rehabil Med. 1989;21:45-53.

15. Guralnik JM, Ferrucci L, Simonsick EM, Salive ME, Wallace RB. Lower-extremity function in persons over the age of 70 years as a predictor of subsequent disability. N Engl J Med. 1995 Mar 2;332(9):556-61. doi: 10.1056/NEJM19950302332 0902.

16. Schache MB, McClelland JA, Webster KE. Lower limb strength following total knee arthroplasty: a systematic review. Knee. 2014 Jan;21(1):12-20. doi: 10.1016/j.knee.2013.08.002. Epub 2013 Aug 15. PMID: 24035477.

17. Judd DL, Wolfe P, LeDoux CV, Hogan C, Dayton MR, Stevens-Lapsley JE. Trajectories of functional performance and muscle strength recovery differ after total knee and total hip replacement: a performance-based, longitudinal study. Int J Rehabil Res. 2019 Sep;42(3):211-216. doi: 10.1097/MRR.0000000000000344.

18. Ashikaga K, Saji M, Takanashi S, Nagayama M, Akashi YJ, Isobe M. Physical performance as a predictor of midterm outcome after mitral valve surgery. Heart Vessels. 2019 Oct;34(10):16651673. doi: 10.1007/s00380-019-01397-y. Epub 2019 Apr 10.

19. Hanada M, Yamauchi K, Miyazaki S, Oyama Y, Yanagita Y, Sato S, Miyazaki T, Nagayasu T, Kozu R. Short-Physical Performance Battery (SPPB) score is associated with postoperative pulmonary complications in elderly patients undergoing lung resection surgery: A prospective multicenter cohort study. Chron Respir Dis. 2020 JanDec;17:1479973120961846. doi: 10.1177/147997 3120961846.

20. Przkora R, Kinsky MP, Fisher SR, Babl C, Heyde CE, Vasilopoulos T, Kaye AD, Volpi E. Functional Improvements Utilizing the Short Physical Performance Battery (SPPB) in the Elderly after Epidural Steroid Injections. Curr Pain Headache Rep. 2019 Feb 22;23(2):14. doi: 10.1007/s11916019-0748-2.

Submitted: February 2, 2021 Accepted for publication: April 1, 2021 